\title{
Quadriceps tendon autograft is becoming increasingly popular in revision $\mathrm{ACL}$ reconstruction
}

\author{
Philipp W. Winkler ${ }^{1,2}$. $\cdot$ Thiago Vivacqua $^{3} \cdot$ Stephan Thomassen $^{3} \cdot$ Lisa Lovse $^{3} \cdot$ Bryson P. Lesniak $^{1}$. \\ Alan M. J. Getgood ${ }^{3} \cdot$ Volker Musahl $^{1}$
}

Received: 29 December 2020 / Accepted: 25 January 2021 / Published online: 16 February 2021

(c) The Author(s) 2021, corrected publication 2021

\begin{abstract}
Purpose To evaluate trends in revision anterior cruciate ligament reconstruction (ACL-R), with emphasis on intra-articular findings, grafts, and concurrent procedures. It was hypothesized that revision ACL-Rs over time show a trend toward increased complexity with increased use of autografts over allografts.

Methods This was a two-center retrospective study including patients undergoing revision ACL-R between 2010 and 2020. Demographic and surgical data including intra-articular findings and concurrent procedures were collected and compared for the time periods 2010-2014 and 2015-2020. All collected variables were compared between three pre-defined age groups ( $<20$ years, 20-30 years, $>30$ years), right and left knees, and males and females. A time series analysis was performed to assess trends in revision ACL-R.

Results This study included 260 patients with a mean age of $26.2 \pm 9.4$ years at the time of the most recent revision ACL-R, representing the first, second, third, and fourth revision ACL-R for 214 (82\%), 35 (14\%), 10 (4\%), and $1(<1 \%)$ patients, respectively. Patients age $>30$ years showed a significantly longer mean time from primary ACL-R to most recent revision ACL-R (11.1 years), compared to patients age $<20$ years $(2.2$ years, $p<0.001)$ and age $20-30$ years $(5.5$ years, $p<0.05$ ). Quadriceps tendon autograft was used significantly more often in 2015-2020 compared to 2010-2014 (49\% vs. $18 \%, p<0.001)$. A high rate of concurrently performed procedures including meniscal repairs (45\%), lateral extra-articular tenodesis (LET; 31\%), osteotomies (13\%), and meniscal allograft transplantations (11\%) was shown. Concurrent LET was associated with intact cartilage and severely abnormal preoperative knee laxity and showed a statistically significant and linear increase over time $(p<0.05)$. Intact cartilage $(41 \%, p<0.05)$, concurrent medial meniscal repairs $(39 \%, p<0.05)$, and LET (35\%, non-significant) were most frequently observed in patients aged $<20$ years.

Conclusion Quadriceps tendon autograft and concurrent LET are becoming increasingly popular in revision ACL-R. Intact cartilage and severely abnormal preoperative knee laxity represent indications for LET in revision ACL-R. The high rate of concurrent procedures observed demonstrates the high surgical demands of revision ACL-R.
\end{abstract}

Level of evidence Level III.

Keywords ACL · Anterior cruciate ligament · Revision · Lateral extra-articular tenodesis · Quadriceps tendon · Allograft

The investigation was performed at the Department of Orthopaedic Surgery, University of Pittsburgh, Pittsburgh, PA, USA and at the Fowler Kennedy Sport Medicine Clinic, University of Western Ontario, London, ON, CAN.

Volker Musahl

musahlv@upmc.edu

1 Department of Orthopaedic Surgery, UPMC Freddie Fu Sports Medicine Center, University of Pittsburgh, 3200 S. Water St., Pittsburgh, PA 15203, USA

2 Department for Orthopaedic Sports Medicine, Klinikum rechts der Isar, Technical University of Munich, Ismaninger Str. 22, 81675 Munich, Germany
Department of Orthopedic Surgery, Fowler Kennedy Sport Medicine Clinic, University of Western Ontario, 3M Centre, 1151 Richmond Street, London, ON N6A 3K7, Canada 


\section{Introduction}

Failure rates of up to $10 \%$ and $30 \%$ after primary and revision anterior cruciate ligament reconstruction (ACL-R), respectively, highlight the clinical relevance of revision ACL-R in daily clinical practice $[12,24,37,42,44,49$, 57-59]. Accordingly, revision ACL-R has been extensively studied in recent years. In particular, the prospective longitudinal Multicenter ACL Revision Study (MARS) provided high-quality data with a high level of evidence for revision ACL-R and identified numerous independent predictors for the outcomes of revision ACL-R [5-8, 23-36, $39,52]$. However, patient enrollment in the MARS cohort ended in 2011 and based on the findings of the MARS the standard of care in revision ACL-R may have changed.

Knee ligament registries represent another valuable source which provide insights into outcomes, failure rates, and descriptive data of revision ACL-R [22, 23]. Despite large sample sizes and generalizability, prospectively collected data from national registries are subject to numerous limitations, including multiple surgeons and surgical techniques, misclassifications, and insufficient knowledge of confounding factors [54]. Consequently, it seems to be difficult to identify changes in practice patterns in revision
ACL-R and the driving causes based on register studies alone.

Therefore, the purpose of this study was to evaluate trends in revision ACL-R based on two surgical sites, with emphasis on intra-articular findings, grafts, and concurrent procedures. It was hypothesized that revision ACL-Rs over time show a trend toward increased complexity in concurrent procedures with increased use of autografts over allografts.

\section{Materials and methods}

Approval for this study was obtained by the institutional review boards of the University of Pittsburgh (No.: STUDY20050226) and the University of Western Ontario (No.: 101533). Given the retrospective design of this study, the need for written informed consent of the included patients was waived.

Patients undergoing revision ACL-R between 2010 and 2020 performed by fellowship-trained knee surgeons at the University of Pittsburgh (VM, BPL) and the University of Western Ontario (AMJG) were screened for eligibility for this retrospective two-center study (Fig. 1). Patients with inflammatory arthritis, previous proximal tibia or distal femur fractures, and incomplete medical records were excluded from the study. Included patients were assigned

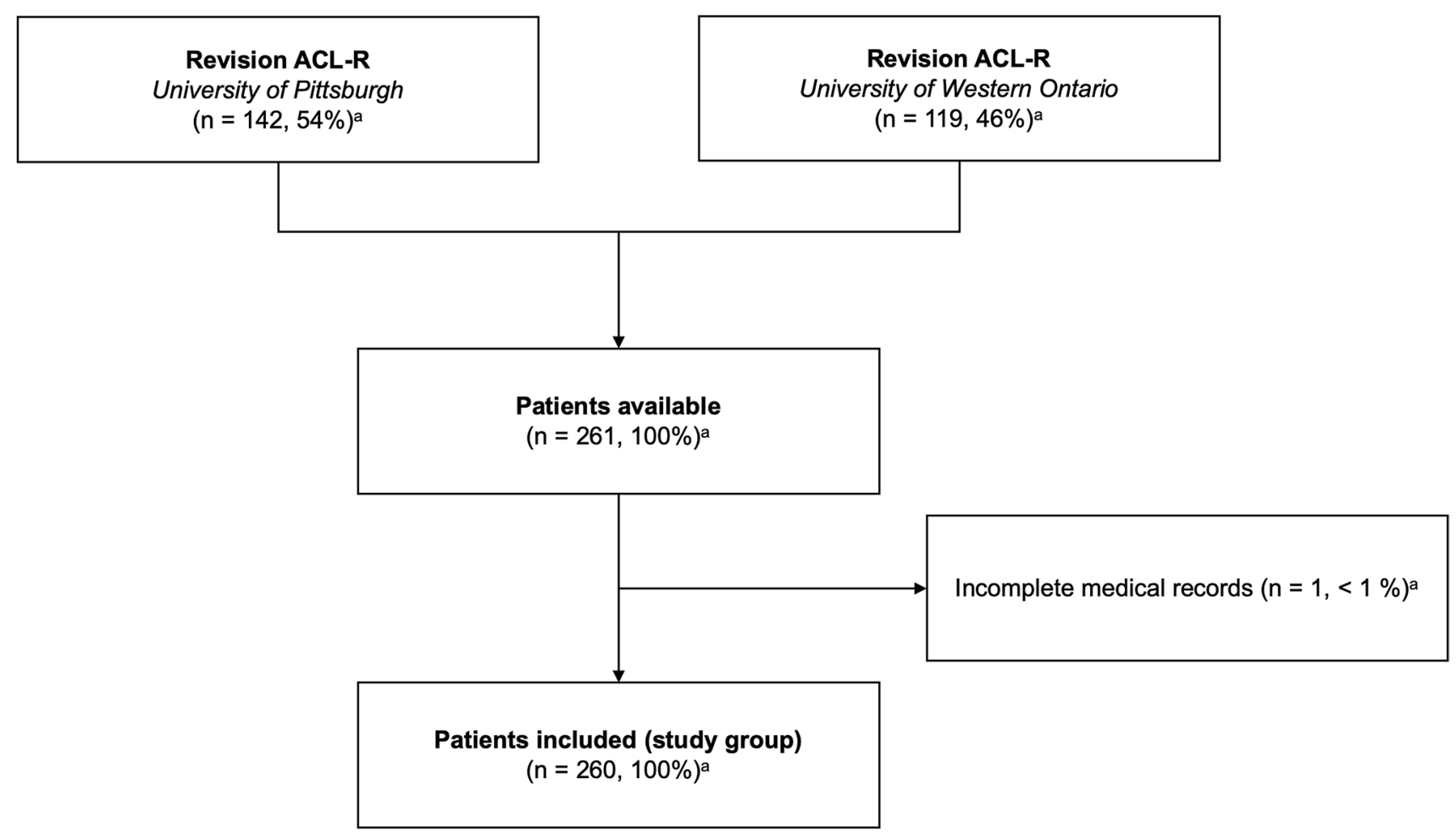

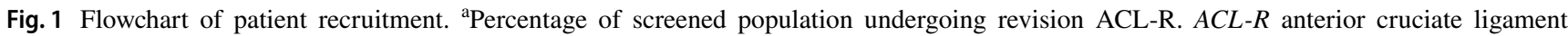
reconstruction 
to one of three pre-defined groups based on the age at the time of the most recent revision ACL-R: $<20$ years, $20-30$ years, $>30$ years. Two time periods, 2010-2014 and 2015-2020, were defined to determine changes in practice patterns over time.

\section{Revision ACL-R and concurrent procedures}

Included patients underwent single-stage or two-stage anatomic single-bundle transportal revision ACL-R by one of the participating fellowship-trained knee surgeons (VM, BPL, AMJG). Graft choice for revision ACL-R was determined by the operating surgeon based on patient history (i.e., prior graft choice(s)), and preoperative (i.e., examination under anesthesia) and intraoperative (i.e., notch size, prior tunnel placement) findings. Grafts used included: ipsilateral and contralateral hamstring tendon, quadriceps tendon, and bone-patellar tendon-bone autografts, and freshfrozen allografts. Concurrent procedures were categorized as follows: repair or reconstruction of the medial collateral ligament (MCL), lateral collateral ligament (LCL), or posterior cruciate ligament (PCL), meniscus surgery, cartilage surgery, osteotomy, and lateral extra-articular tenodesis (LET). Meniscus surgery was categorized as meniscal repair, partial meniscectomy, and meniscal allograft transplantation (MAT). Cartilage surgery included microfracture, osteochondral autograft and allograft transplantation, matrix-induced autologous chondrocyte implantation, and application of particulated juvenile articular cartilage [10]. The osteotomy types performed included: medial open wedge, medial closed wedge, lateral closed wedge, and anterior closed wedge high tibial osteotomy and medial closed wedge distal femoral osteotomy.

\section{Demographic and surgical data}

A review of medical records was performed by one observer at each center (PWW, TV) between March and October 2020 to collect demographic and surgical data. Demographic data collected included sex, affected knee, body-mass-index (BMI), age at the time of most recent revision ACL-R, and the prevalence of contralateral anterior cruciate ligament (ACL) injuries. Surgical data from the most recent revision ACL-R collected included staging (single-stage vs. twostage revision ACL-R), grade of preoperative Lachman and pivot-shift tests during examination under anesthesia, intraarticular findings, graft type, graft diameter, femoral and tibial graft fixation technique, and concurrently performed intra- and extra-articular surgical procedures. Furthermore, the number of revision ACL-R performed was documented. Preoperative Lachman and pivot-shift tests were graded as normal, nearly normal, abnormal, and severely abnormal according to the International Knee Documentation
Committee (IKDC) Knee Examination Form. Cartilage conditions were evaluated according to the International Cartilage Repair Society (ICRS) grading system and were classified as intact cartilage (ICRS grade 0), low-grade lesion (ICRS grade 1 and grade 2), and high-grade lesion (ICRS grade 3 and grade 4). Meniscal lesions observed were categorized by location (medial, lateral) and type (horizontal, longitudinal, radial, ramp, complex, root, status post meniscal repair, status post partial meniscectomy). Four techniques of femoral graft fixation (suspensory, interference screw, hybrid, over-the-top) and three techniques of tibial graft fixation (suspensory, interference screw, hybrid) were defined. Hybrid graft fixation represents a combination of suspensory and interference screw graft fixation.

\section{Radiographic data}

The medial and lateral posterior tibial slope (PTS), the anterior-posterior lateral femoral condyle length, and the lateral femoral condyle depth were measured on strict lateral radiographs, as previously described $[41,45]$. Based on the lateral femoral condyle length and depth, the lateral femoral condyle ratio (LFCR) was calculated [45]. To avoid measurement inaccuracies, lateral radiographs with $>6 \mathrm{~mm}$ posterior femoral condyle overlap were excluded for the measurement of the medial and lateral PTS, and the lateral femoral condyle length and depth [45].

All measurements were performed by one observer at each center (PWW, TV). Inter- and intra-rater reliability of measurements between and within the two observers was evaluated based on 10 randomly selected patients. Intraclass correlation coefficients (ICC) were calculated and revealed good to excellent intra-rater (ICC 0.895-0.987) and moderate to excellent inter-rater (ICC $0.725-0.978$ ) reliability of measurements.

\section{Statistical analysis}

Categorical variables were expressed as number of patients and percentage of the corresponding group. Continuous variables were reported as mean \pm standard deviation and range. Group comparisons with two independent variables (male vs. female; right vs. left; 2010-2014 vs. 2015-2020) were performed by the Chi-squared test for categorical variables and by the unpaired $t$ test or Mann-Whitney $U$ test for continuous variables. Group comparisons for the three pre-defined age groups ( $<20$ years vs. $20-30$ years vs. $>30$ years) were performed by the Chi-squared test (followed by a post hoc test with Bonferroni corrected $p$ values) for categorical variables and by a one-way analysis of variance or the Kruskal-Wallis test (followed by post hoc testing) for continuous variables. To assess the trend of concurrently performed procedures over the 10 -year period, 
a time series analysis (i.e., linear regression model) was performed. The percentage of combined revision ACL-R (ACL-R + meniscus surgery, ACL-R + LET, ACL-R + osteotomy) among all revision ACL-Rs performed annually represented the dependent variables, while the corresponding year represented the independent variable [2]. Given the descriptive design of this study and that all patients available were included, an a priori sample size calculation was not conducted. SPSS software version 26.0 (IBM-SPSS, New York, USA) was used for statistical analysis. The level of significance was defined as $p<0.05$.

\section{Results}

Out of 261 patients screened for eligibility, $1 \quad(<1 \%)$ patient had to be excluded due to incomplete medical records (Fig. 1). Thus, 260 patients with a mean age of $26.2 \pm 9.4$ years (range 13-58 years) at the time of the most recent revision ACL- $\mathrm{R}$, were included in this study. The mean time from primary ACL-R to the most recent revision ACL-R was $6.1 \pm 6.0$ years (range $0.2-40.0$ years) for the total study group. The most recent revision ACL-R represented a single-stage procedure in 223 (86\%) patients and was the first, second, third, and fourth revision ACL-R for $214(82 \%), 35(14 \%), 10(4 \%)$, and 1 (<1\%) patients, respectively. Concomitant MCL, PCL, and LCL injuries were observed in $13(5 \%), 5(2 \%)$, and 4 (2\%) patients, respectively.

\section{Sex and laterality}

Statistically significantly more MCL injuries were observed in males compared to females ( $8 \%$ vs. $1 \%, p<0.05)$. Females were found to have statistically significantly more contralateral ACL injuries ( $18 \%$ vs. $8 \%, p<0.05)$ and a higher LFCR (0.66 vs. $0.64, p<0.001)$ compared to males. All other variables showed no statistically significant difference between males and females. No statistically significant differences were found between right and left knees for all variables analyzed.

\section{Age groups}

\section{Demographic, surgical, and radiographic data}

A detailed overview is shown in Table 1. The mean time from primary ACL-R to the most recent revision ACL-R was $2.2 \pm 1.6$ years, $5.5 \pm 3.5$ years, and $11.1 \pm 8.6$ years for patients age $<20$ years, 20-30 years, and $>30$ years, respectively, representing a statistically significant difference between all three pre-defined age groups $(<20$ years vs. $20-30$ years, $p<0.001 ;<20$ years vs. $>30$ years, $p<0.001$;
20-30 years vs. $>30$ years, $p<0.05)$. There was a statistically significant association between the graft used for the most recent revision ACL-R and the three age groups $(p<0.001)$.

\section{Intra-articular findings}

A detailed overview is shown in Table 2. There were statistically significantly more patients with intact cartilage in the age group $<20$ years compared to the other two age groups $(p<0.05)$. Patients age $>30$ years had statistically significantly less intact cartilage at the medial femoral condyle $(p<0.001)$, lateral tibial plateau $(p<0.001)$, trochlea $(p<0.05)$, and patella $(p<0.001)$. Patients age $>30$ years had also statistically significantly more high-grade cartilage lesions at the medial femoral condyle $(p<0.001)$ and patients age $<20$ years had significantly more intact cartilage at the medial tibial plateau $(p<0.001)$.

\section{Concurrent procedures}

A detailed overview is shown in Table 3. Statistically significantly more medial meniscal repairs were performed in the age group $<20$ years $(p<0.05)$. Concurrent osteotomies were performed significantly more often in the age group $>30$ years $(23 \%, p<0.05)$. In patients with concurrent LET (31\%), significantly more intact cartilage was observed at the medial femoral condyle $(64 \%$ vs. $38 \%, p<0.001)$, lateral femoral condyle $(78 \%$ vs. $61 \%, p<0.05)$, medial tibial plateau $(80 \%$ vs. $56 \%, p<0.001)$, lateral tibial plateau ( $84 \%$ vs. $63 \%, p<0.05)$, and at the trochlea (94\% vs. $77 \%$, $p<0.05)$ compared to patients without concurrent LET. In addition, preoperative Lachman and pivot-shift tests were significantly more frequently classified as severely abnormal in patients with than without concurrent LET (Lachman, $42 \%$ vs. $21 \%, p<0.05$; pivot-shift, $42 \%$ vs. $19 \%, p<0.001)$.

\section{Trends over time}

Revision ACL-R was performed in 66 patients (58\% male) with a mean age of $25.7 \pm 8.1$ years (range $15-48$ years) between 2010 and 2014 and in 194 patients (55\% male) with a mean age of $26.4 \pm 9.8$ years (range $13-58$ years) between 2015 and 2020. Quadriceps tendon autograft was used significantly more often in 2015-2020 compared to $2010-2014$ (49\% vs. $18 \%, p<0.001)$. More medial [27\% vs. $23 \%$, non-significant (n.s.)] and lateral meniscal repairs ( $21 \%$ vs. $14 \%$, n.s.), osteotomies (13\% vs. $11 \%$, n.s.), and LETs (34\% vs. $23 \%$, n.s.) were performed in 2015-2020 compared to 2010-2014. Time series analysis revealed a statistically significant and positive linear relationship between the time period (2010-2020) and the percentage of combined revision ACL-R + LET among 
Table 1 Demographic, surgical, and radiographic data of the total study group and the three pre-defined age groups

\begin{tabular}{|c|c|c|c|c|c|}
\hline \multirow[t]{2}{*}{ Variables } & \multirow[t]{2}{*}{ Total study group } & \multicolumn{3}{|l|}{ Age group ${ }^{a}$} & \multirow[t]{2}{*}{$p$ value } \\
\hline & & $<20$ years & $20-30$ years & $>30$ years & \\
\hline Number of patients $(n)$ & 260 & 71 & 120 & 69 & - \\
\hline Age, ${ }^{\mathrm{a}}$ (years) & $26.2 \pm 9.4(13-58)$ & $17.4 \pm 1.5(13-19)$ & $23.9 \pm 3.0(20-30)$ & $39.4 \pm 6.9(31-58)$ & $<0.001 *$ \\
\hline BMI, $\left[\mathrm{kg} / \mathrm{m}^{2}\right]$ & $26.9 \pm 5.0(19.0-48.0)$ & $25.4 \pm 3.7(20.0-40.0)$ & $26.2 \pm 4.8(19.0-45.3)$ & $29.8 \pm 5.5(21.2-48.0)$ & $<0.001 *$ \\
\hline $\begin{array}{l}\text { Primary ACL-R to most recent } \\
\text { revision ACL-R, [years] }\end{array}$ & $6.1 \pm 6.0(0.2-40.0)$ & $2.2 \pm 1.6(0.5-6.8)$ & $5.5 \pm 3.5(0.2-14.8)$ & $11.1 \pm 8.6(0.3-40.0)$ & $<0.001 *$ \\
\hline Males, $n(\%)$ & $144(55 \%)$ & $34(48 \%)$ & $65(54 \%)$ & $45(65 \%)$ & n.s \\
\hline Right knee, $n(\%)$ & $123(47 \%)$ & $35(49 \%)$ & $53(44 \%)$ & $35(51 \%)$ & n.s \\
\hline Number of revision ACL-R & & & & & n.s \\
\hline First, $n(\%)$ & $214(82 \%)$ & $66(93 \%)$ & $91(76 \%)$ & $57(83 \%)$ & \\
\hline Second, $n(\%)$ & $35(14 \%)$ & $5(7 \%)$ & $21(18 \%)$ & $9(13 \%)$ & \\
\hline Third, $n(\%)$ & $10(4 \%)$ & $0(0 \%)$ & $7(6 \%)$ & $3(4 \%)$ & \\
\hline Fourth, $n(\%)$ & $1(<1 \%)$ & $0(0 \%)$ & $1(1 \%)$ & $0(0 \%)$ & \\
\hline Graft $^{\mathrm{a}}$ & & & & & $<0.001 *$ \\
\hline Hamstring, $n(\%)$ & $8(3 \%)$ & $0(0 \%)$ & $3(3 \%)$ & $5(7 \%)$ & \\
\hline Quadriceps, $n(\%)$ & $106(41 \%)$ & $34(48 \%)$ & $52(43 \%)$ & $20(29 \%)$ & \\
\hline $\mathrm{BPTB}, n(\%)$ & $88(34 \%)$ & $31(44 \%)$ & $42(35 \%)$ & $15(22 \%)$ & \\
\hline Allograft, $n(\%)$ & $55(21 \%)$ & $6(9 \%)$ & $21(18 \%)$ & $28(41 \%)$ & \\
\hline Hamstring cont., $n(\%)$ & $1(<1 \%)$ & $0(0 \%)$ & $0(0 \%)$ & $1(1 \%)$ & \\
\hline BPTB cont., $n(\%)$ & $2(1 \%)$ & $0(0 \%)$ & $2(2 \%)$ & $0(0 \%)$ & \\
\hline Graft diameter, ${ }^{\mathrm{a}}[\mathrm{mm}]$ & $9.6 \pm 0.7(7.5-12.0)$ & $9.7 \pm 0.6(8.0-11.0)$ & $9.6 \pm 0.7(8.0-12.0)$ & $9.4 \pm 0.7(7.5-11.0)$ & $<0.05^{*}$ \\
\hline Femoral graft fixation $^{a}$ & & & & & n.s \\
\hline Suspensory, $n(\%)$ & $179(69 \%)$ & $48(68 \%)$ & $83(69 \%)$ & $48(70 \%)$ & \\
\hline Interference, $n(\%)$ & $38(15 \%)$ & $11(16 \%)$ & $16(13 \%)$ & $11(16 \%)$ & \\
\hline Over-the-top, $n(\%)$ & $38(15 \%)$ & $12(17 \%)$ & $16(13 \%)$ & $10(15 \%)$ & \\
\hline Hybrid, $n(\%)$ & $4(2 \%)$ & $0(0 \%)$ & $4(3 \%)$ & $0(0 \%)$ & \\
\hline N/A, $n(\%)$ & $1(<1 \%)$ & $0(0 \%)$ & $1(1 \%)$ & $0(0 \%)$ & \\
\hline Tibial graft fixation ${ }^{a}$ & & & & & n.s \\
\hline Suspensory, $n(\%)$ & $66(25 \%)$ & $21(30 \%)$ & $29(24 \%)$ & $16(23 \%)$ & \\
\hline Interference, $n(\%)$ & $160(62 \%)$ & $44(62 \%)$ & $71(59 \%)$ & $45(65 \%)$ & \\
\hline Hybrid, $n(\%)$ & $32(12 \%)$ & $6(9 \%)$ & $18(15 \%)$ & $8(12 \%)$ & \\
\hline N/A, $n(\%)$ & $2(1 \%)$ & $0(0 \%)$ & $2(2 \%)$ & $0(0 \%)$ & \\
\hline $\begin{array}{l}\text { Contralateral ACL injury, } n \\
(\%)\end{array}$ & $33(13 \%)$ & $12(17 \%)$ & $13(11 \%)$ & $8(12 \%)$ & n.s \\
\hline $\mathrm{LFCR}^{\mathrm{b}}{ }^{\mathrm{b}}[-]$ & $0.65 \pm 0.04(0.52-0.84)$ & $0.64 \pm 0.05(0.53-0.75)$ & $0.65 \pm 0.05(0.55-0.84)$ & $0.66 \pm 0.04(0.52-0.75)$ & n.s \\
\hline Medial PTS, ${ }^{\mathrm{b}}\left[{ }^{\circ}\right]$ & $10.2 \pm 3.3(2.0-19.0)$ & $9.9 \pm 3.7(4.0-19.0)$ & $10.8 \pm 3.3(2.0-18.0)$ & $9.4 \pm 2.8(3.2-15.0)$ & $<0.05^{*}$ \\
\hline Lateral PTS, ${ }^{\mathrm{b}}\left[{ }^{\circ}\right]$ & $9.5 \pm 3.8(1.0-22.0)$ & $9.0 \pm 4.3(1.0-22.0)$ & $10.3 \pm 3.7(3.0-20.0)$ & $8.6 \pm 3.4(2.0-18.0)$ & $<0.05^{*}$ \\
\hline
\end{tabular}

Categorical variables are expressed as mean (corresponding percentage). Continuous variables are expressed as mean \pm standard deviation (range)

$A C L$ anterior cruciate ligament, $A C L-R$ ACL reconstruction, $B M I$ body mass index, BPTB bone-patellar tendon-bone, cont. contralateral, $L F C R$ lateral femoral condyle ratio, N/A not available, n.s. non-significant, PTS posterior tibial slope

*Statistically significant difference $(p<0.05)$

${ }^{a}$ At most recent revision ACL-R

${ }^{\mathrm{b}}$ Data available for 214 patients (82\% of study group)

revision ACL-R performed annually ( $p<0.05$; adjusted $R^{2}=0.658$; Fig. 2 ). No statistically significant relationship could be observed between the proportion of other concurrent procedures (meniscal repair, meniscectomy, MAT, osteotomy) among the revision ACL-Rs performed annually. 
Table 2 Intra-articular findings at most recent revision ACL-R of the total study group and the three pre-defined age groups

\begin{tabular}{|c|c|c|c|c|c|}
\hline \multirow[t]{2}{*}{ Variables } & \multirow[t]{2}{*}{ Total study group } & \multicolumn{3}{|l|}{ Age group } & \multirow[t]{2}{*}{$p$ value } \\
\hline & & $<20$ years & $20-30$ years & $>30$ years & \\
\hline Meniscus lesion & & & & & n.s \\
\hline None, $n(\%)$ & $39(15 \%)$ & $12(17 \%)$ & $20(17 \%)$ & $7(10 \%)$ & \\
\hline Medial, $n(\%)$ & $83(32 \%)$ & $17(24 \%)$ & $39(33 \%)$ & $27(39 \%)$ & \\
\hline Lateral, $n(\%)$ & $48(19 \%)$ & $13(18 \%)$ & $18(15 \%)$ & $17(25 \%)$ & \\
\hline Both, $n(\%)$ & $90(35 \%)$ & $29(41 \%)$ & $43(36 \%)$ & $18(26 \%)$ & \\
\hline Medial meniscal tear type & & & & & n.s \\
\hline None, $n(\%)$ & $87(33 \%)$ & $25(35 \%)$ & $38(32 \%)$ & $24(35 \%)$ & \\
\hline Horizontal, $n(\%)$ & $2(1 \%)$ & $0(0 \%)$ & $1(1 \%)$ & $1(1 \%)$ & \\
\hline Longitudinal, $n(\%)$ & $59(23 \%)$ & $19(27 \%)$ & $29(24 \%)$ & $11(16 \%)$ & \\
\hline Radial, $n(\%)$ & $7(3 \%)$ & $0(0 \%)$ & $5(4 \%)$ & $2(3 \%)$ & \\
\hline Ramp, $n(\%)$ & $21(8 \%)$ & $11(16 \%)$ & $5(4 \%)$ & $5(7 \%)$ & \\
\hline Complex, $n(\%)$ & $31(12 \%)$ & $5(7 \%)$ & $17(14 \%)$ & $9(13 \%)$ & \\
\hline Root, $n(\%)$ & $5(2 \%)$ & $0(0 \%)$ & $3(3 \%)$ & $2(3 \%)$ & \\
\hline s/p partial ME, $n(\%)$ & $41(16 \%)$ & $8(11 \%)$ & $19(16 \%)$ & $14(20 \%)$ & \\
\hline $\mathrm{s} / \mathrm{p}$ meniscal repair, $n(\%)$ & $7(3 \%)$ & $3(4 \%)$ & $3(3 \%)$ & $1(1 \%)$ & \\
\hline Lateral meniscal tear type & & & & & n.s \\
\hline None, $n(\%)$ & $122(47 \%)$ & $29(41 \%)$ & $59(49 \%)$ & $34(49 \%)$ & \\
\hline Horizontal, $n(\%)$ & $4(2 \%)$ & $1(1 \%)$ & $2(2 \%)$ & $1(1 \%)$ & \\
\hline Longitudinal, $n(\%)$ & $30(12 \%)$ & $8(11 \%)$ & $14(12 \%)$ & $8(12 \%)$ & \\
\hline Radial, $n(\%)$ & $11(4 \%)$ & $2(3 \%)$ & $4(3 \%)$ & $5(7 \%)$ & \\
\hline Complex, $n(\%)$ & $11(4 \%)$ & $5(7 \%)$ & $4(3 \%)$ & $2(3 \%)$ & \\
\hline Root, $n(\%)$ & $40(15 \%)$ & $16(23 \%)$ & $16(13 \%)$ & $8(12 \%)$ & \\
\hline s/p partial ME, $n(\%)$ & $30(12 \%)$ & $8(11 \%)$ & $15(13 \%)$ & $7(10 \%)$ & \\
\hline s/p meniscal repair, $n(\%)$ & $11(4 \%)$ & $2(3 \%)$ & $6(5 \%)$ & $3(4 \%)$ & \\
\hline N/A, $n(\%)$ & $1(<1 \%)$ & $0(0 \%)$ & $0(0 \%)$ & $1(1 \%)$ & \\
\hline Cartilage lesion, $n(\%)$ & $190(73 \%)$ & $42(59 \%)$ & $91(76 \%)$ & $57(83 \%)$ & $<0.05^{*}$ \\
\hline Cartilage medial femoral condyle & & & & & $<0.001^{*}$ \\
\hline Intact, $n(\%)$ & $120(46 \%)$ & $42(59 \%)$ & $57(48 \%)$ & $21(30 \%)$ & \\
\hline Low-grade, $n(\%)$ & $102(39 \%)$ & $23(32 \%)$ & $52(43 \%)$ & $27(39 \%)$ & \\
\hline High-grade, $n(\%)$ & $38(15 \%)$ & $6(9 \%)$ & $11(9 \%)$ & $21(30 \%)$ & \\
\hline Cartilage medial tibial plateau & & & & & $<0.05^{*}$ \\
\hline Intact, $n(\%)$ & $165(63 \%)$ & $57(80 \%)$ & $73(61 \%)$ & $35(51 \%)$ & \\
\hline Low-grade, $n(\%)$ & $87(34 \%)$ & $14(20 \%)$ & $43(36 \%)$ & $30(43 \%)$ & \\
\hline High-grade, $n(\%)$ & $8(3 \%)$ & $0(0 \%)$ & $4(3 \%)$ & $4(6 \%)$ & \\
\hline Cartilage lateral femoral condyle & & & & & n.s \\
\hline Intact, $n(\%)$ & $172(66 \%)$ & $48(68 \%)$ & $82(68 \%)$ & $42(61 \%)$ & \\
\hline Low-grade, $n(\%)$ & $56(22 \%)$ & $15(21 \%)$ & $23(19 \%)$ & $18(26 \%)$ & \\
\hline High-grade, $n(\%)$ & $32(12 \%)$ & $8(3 \%)$ & $15(6 \%)$ & $9(4 \%)$ & \\
\hline Cartilage lateral tibial plateau & & & & & $<0.05^{*}$ \\
\hline Intact, $n(\%)$ & $181(70 \%)$ & $53(75 \%)$ & $91(76 \%)$ & $37(54 \%)$ & \\
\hline Low-grade, $n(\%)$ & $62(24 \%)$ & $15(21 \%)$ & $23(19 \%)$ & $24(35 \%)$ & \\
\hline High-grade, $n(\%)$ & $17(6 \%)$ & $3(4 \%)$ & $6(5 \%)$ & $8(12 \%)$ & \\
\hline Cartilage trochlea & & & & & $<0.05^{*}$ \\
\hline Intact, $n(\%)$ & $214(82 \%)$ & $64(90 \%)$ & $101(84 \%)$ & $49(71 \%)$ & \\
\hline Low-grade, $n(\%)$ & $27(10 \%)$ & $7(10 \%)$ & $9(8 \%)$ & $11(16 \%)$ & \\
\hline High-grade, $n(\%)$ & $19(7 \%)$ & $0(0 \%)$ & $10(8 \%)$ & $9(13 \%)$ & \\
\hline Cartilage patella & & & & & $<0.05^{*}$ \\
\hline Intact, $n(\%)$ & $199(77 \%)$ & $60(85 \%)$ & $97(81 \%)$ & $42(61 \%)$ & \\
\hline Low-grade, $n(\%)$ & $54(21 \%)$ & $11(15 \%)$ & $21(18 \%)$ & $22(32 \%)$ & \\
\hline High-grade, $n(\%)$ & $7(3 \%)$ & $0(0 \%)$ & $2(2 \%)$ & $5(7 \%)$ & \\
\hline
\end{tabular}

Categorical variables are expressed as mean (corresponding percentage)

$A C L-R$ anterior cruciate ligament reconstruction, $M E$ meniscectomy, N/A not available, n.s. non-significant, $s / p$ status post 
Table 2 (continued)

Table 3 Concurrent procedures at most recent revision ACL-R of the total study group and the three pre-defined age groups
*Statistically significant difference $(p<0.05)$

\begin{tabular}{|c|c|c|c|c|c|}
\hline \multirow[t]{2}{*}{ Variables } & \multirow[t]{2}{*}{ Total study group } & \multicolumn{3}{|l|}{ Age group } & \multirow[t]{2}{*}{$p$ value } \\
\hline & & $<20$ years & $20-30$ years & $>30$ years & \\
\hline Medial meniscus treatment & & & & & $<0.05^{*}$ \\
\hline No treatment, $n(\%)$ & $117(45 \%)$ & $31(44 \%)$ & $52(43 \%)$ & $34(49 \%)$ & \\
\hline Repair, $n(\%)$ & $68(26 \%)$ & $28(39 \%)$ & $25(21 \%)$ & $15(22 \%)$ & \\
\hline Partial ME, $n(\%)$ & $51(20 \%)$ & $9(13 \%)$ & $29(24 \%)$ & $13(19 \%)$ & \\
\hline MAT, $n(\%)$ & $24(9 \%)$ & $3(4 \%)$ & $14(12 \%)$ & $7(10 \%)$ & \\
\hline Lateral meniscus treatment & & & & & n.s \\
\hline No treatment, $n(\%)$ & $170(65 \%)$ & $43(61 \%)$ & $80(67 \%)$ & $47(68 \%)$ & \\
\hline Repair, $n(\%)$ & $49(19 \%)$ & $16(23 \%)$ & $21(18 \%)$ & $12(17 \%)$ & \\
\hline Partial ME, $n(\%)$ & $37(14 \%)$ & $11(16 \%)$ & $16(13 \%)$ & $10(15 \%)$ & \\
\hline MAT, $n(\%)$ & $4(2 \%)$ & $1(1 \%)$ & $3(3 \%)$ & $0(0 \%)$ & \\
\hline PCL treatment & & & & & n.s \\
\hline None, $n(\%)$ & $258(99 \%)$ & $70(99 \%)$ & $120(100 \%)$ & $68(99 \%)$ & \\
\hline Repair, $n(\%)$ & $0(0 \%)$ & $0(0 \%)$ & $0(0 \%)$ & $0(0 \%)$ & \\
\hline Reconstruction, $n(\%)$ & $2(1 \%)$ & $1(1 \%)$ & $0(0 \%)$ & $1(1 \%)$ & \\
\hline MCL treatment & & & & & n.s \\
\hline None, $n(\%)$ & $253(97 \%)$ & $71(100 \%)$ & $117(98 \%)$ & $65(94 \%)$ & \\
\hline Repair, $n(\%)$ & $0(0 \%)$ & $0(0 \%)$ & $0(0 \%)$ & $0(0 \%)$ & \\
\hline Reconstruction, $n(\%)$ & $7(3 \%)$ & $0(0 \%)$ & $3(3 \%)$ & $4(6 \%)$ & \\
\hline LCL treatment & & & & & n.s \\
\hline None, $n(\%)$ & $259(100 \%)$ & $70(99 \%)$ & $120(100 \%)$ & $69(100 \%)$ & \\
\hline Repair, $n(\%)$ & $0(0 \%)$ & $0(0 \%)$ & $0(0 \%)$ & $0(0 \%)$ & \\
\hline Reconstruction, $n(\%)$ & $1(<1 \%)$ & $1(1 \%)$ & $0(0 \%)$ & $0(0 \%)$ & \\
\hline Osteotomy & & & & & $<0.05^{*}$ \\
\hline None, $n(\%)$ & $227(87 \%)$ & $67(94 \%)$ & $107(89 \%)$ & $53(77 \%)$ & \\
\hline HTO slope reducing & $13(5 \%)$ & $3(4 \%)$ & $6(5 \%)$ & $4(6 \%)$ & \\
\hline HTO-MOW, $n(\%)$ & $13(5 \%)$ & $0(0 \%)$ & $3(3 \%)$ & $10(15 \%)$ & \\
\hline HTO-LCW, $n(\%)$ & $3(1 \%)$ & $0(0 \%)$ & $2(2 \%)$ & $1(1 \%)$ & \\
\hline DFO-MCW, $n(\%)$ & $3(1 \%)$ & $1(1 \%)$ & $1(1 \%)$ & $1(1 \%)$ & \\
\hline HTO-MCW, $n(\%)$ & $1(<1 \%)$ & $0(0 \%)$ & $1(1 \%)$ & $0(0 \%)$ & \\
\hline $\begin{array}{l}\text { Lateral extra-articular } \\
\text { tenodesis, } n(\%)\end{array}$ & $81(31 \%)$ & $25(35 \%)$ & $41(34 \%)$ & $15(22 \%)$ & n.s \\
\hline Cartilage surgery & & & & & n.s \\
\hline None, $n(\%)$ & $237(91 \%)$ & $66(93 \%)$ & $107(89 \%)$ & $64(93 \%)$ & \\
\hline OATS autograft, $n(\%)$ & $5(2 \%)$ & $2(3 \%)$ & $2(2 \%)$ & $1(1 \%)$ & \\
\hline OATS allograft, $n(\%)$ & $2(1 \%)$ & $0(0 \%)$ & $2(2 \%)$ & $0(0 \%)$ & \\
\hline Microfracture, $n(\%)$ & $14(5 \%)$ & $3(4 \%)$ & $8(7 \%)$ & $3(4 \%)$ & \\
\hline MACI, $n(\%)$ & $1(<1 \%)$ & $0(0 \%)$ & $1(1 \%)$ & $0(0 \%)$ & \\
\hline PJAC, $n(\%)$ & $1(<1 \%)$ & $0(0 \%)$ & $0(0 \%)$ & $1(1 \%)$ & \\
\hline
\end{tabular}

Categorical variables are expressed as mean (corresponding percentage)

$A C L-R$ anterior cruciate ligament reconstruction, DFO distal femoral osteotomy, HTO high tibial osteotomy, $L C L$ lateral collateral ligament, $L C W$ lateral closed wedge, $M A C I$ matrix-induced autologous chondrocyte implantation, $M A T$ meniscal allograft transplantation, $M C L$ medial collateral ligament, $M C W$ medial closed wedge, $M E$ meniscectomy, $M O W$ medial open wedge, n.s. non-significant, OATS osteochondral transplantation, $P C L$ posterior cruciate ligament, $P J A C$ particulated juvenile articular cartilage

*Statistically significant difference $(p<0.05)$ 


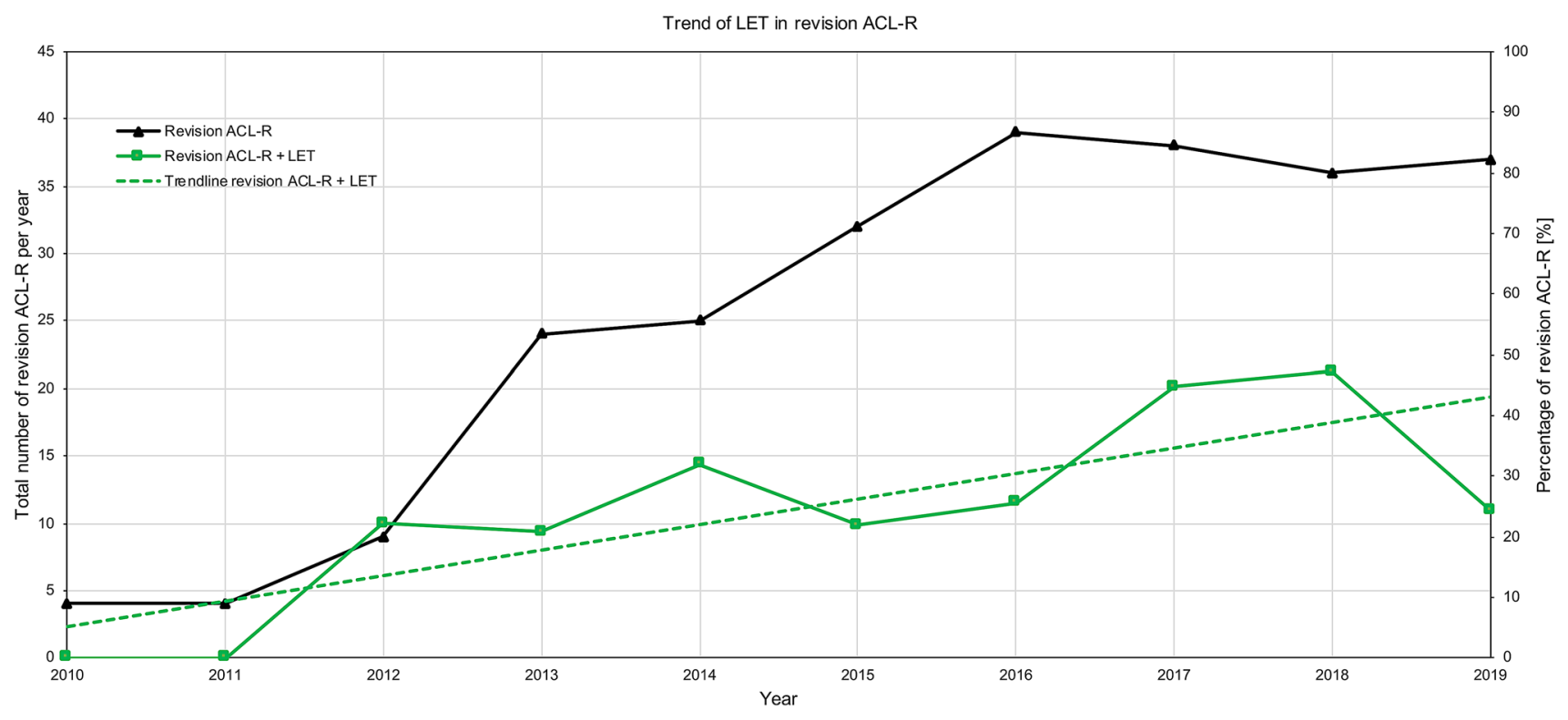

Fig. 2 Trend of combined revision ACL-R + LET. The black line represents the total number of revision ACL-Rs performed annually (left scale). The green line represents combined revision ACL-Rs + LET as a percentage of the total number of revision ACL-Rs performed annually (right scale). The dotted green line represents the trendline of combined revision ACL-Rs + LET, indicating a statistically significant linear increase over time ( $p<0.05$; adjusted $\left.R^{2}=0.658\right)$. $A C L-R$ anterior cruciate ligament reconstruction, $L E T$ lateral extra-articular tenodesis

\section{Discussion}

The most important finding of this study was that quadriceps tendon autografts and concurrent LET are becoming increasingly popular in revision ACL-R, especially in younger patients. Young patient age was also associated with less time between primary ACL-R and the most recent revision ACL-R, more medial meniscal repairs, and higher rates of intact cartilage. In addition, a high and growing rate of concurrent meniscal repairs (45\%), LET (31\%), and osteotomies $(13 \%)$ was observed, underscoring the high surgical demands of revision ACL-R. Moreover, LET in revision ACL-R was associated with intact cartilage and severely abnormal preoperative knee laxity.

Graft choice and availability in the setting of revision ACL-R are different than for primary ACL-R, especially if multiple graft failures have already occurred. Previous research highlighted the importance of graft choice in the setting of revision ACL-R, as it was demonstrated that graft choice represents a significant predictor for functional outcomes and graft failure rates at 2-year follow-up [24]. Between 2006 and 2011, 1205 patients undergoing revision ACL-R were enrolled in the MARS cohort. Allografts were used in $49 \%$, autografts in $48 \%$, and a combination of allografts and autografts in 3\% for revision ACL-R. Regression analyses revealed that the use of autografts in the setting of revision ACL-R resulted in improved patient reported outcomes, sports function, and a significantly decreased risk of subsequent graft failure compared to the use of allografts [24]. The clinical impact of previous research is reflected in the findings of this study, where a notable decrease in the use of allografts (21\%) and an increase in the use of autografts (79\%) compared to the MARS cohort was observed. Looking at European countries, where allografts are often not readily available, the different types of autografts used for revision ACL-R can be evaluated [23]. The Danish Registry for Knee Ligament Reconstructions, the Norwegian Knee Ligament Registry, and the revision ACL-R cohort of the Société Française d'Arthroscopie showed that the most frequently used types of autografts for revision ACL-R were hamstring tendon (39-56\%), followed by bone-patellar tendon-bone (28-56\%), and quadriceps tendon $(2 \%)$ [22, 23]. In this study, hamstring tendon (ipsilateral + contralateral), bone-patellar tendon-bone (ipsilateral + contralateral), and quadriceps tendon autografts were used in $4 \%, 35 \%$, and $41 \%$ of patients, respectively. While the percentage of bonepatellar tendon-bone autografts used is consistent with previous reports, in this study, hamstring and quadriceps tendon autografts are strikingly under- and over-represented, respectively. An almost eightfold increase in the use of quadriceps tendon autograft was observed in the second (2015-2020) compared to the first (2010-2014) half of the observation period of this study. Accordingly, quadriceps tendon autograft represents the currently most frequently used graft for revision ACL- $\mathrm{R}$ in the two participating centers. The reason for the shift in autograft types used for revision ACL-R may 
be due to the emerging evidence of increased failure rates for hamstring tendon autografts and decreased failure rates for quadriceps tendon autografts in ACL-R [12, 38, 46, 49].

Failures in ACL-R may also be caused by persistent rotatory knee laxity. Improved anatomical and biomechanical knowledge of the antero-lateral structures of the knee has increased the awareness of antero-lateral rotatory knee laxity as a cause of ACL graft failure [11, 18, 19]. A randomized controlled trial has demonstrated statistically significantly less ACL graft failures in patients undergoing primary ACL-R + LET (4\%) compared to patients undergoing isolated hamstring tendon autograft primary ACL-R (11\%) [12]. Revision ACL-R is often considered the primary indication for an additional LET, with good mid-term outcomes and low failure rates reported $[1,16,48]$. In this study, $31 \%$ of patients underwent concurrent LET, with the majority of patients receiving LET being $<30$ years old. Patients undergoing concurrent LET were characterized by severely abnormal preoperative knee laxity and low cartilage wear.

Numerous studies have demonstrated the chondroprotective and stabilizing role of the menisci, underscoring their vital role in maintaining normal knee kinematics and function $[3,20,40]$. As a result, meniscal repairs are increasing and high success rates have been reported in the setting of revision ACL-R [26, 43]. In one study, $18 \%$ of patients undergoing revision ACL-R underwent concurrent meniscal repair, with more than two-thirds of repairs accounting for the medial meniscus and an overall failure rate of meniscal repairs of 9\% [26]. Similarly, in the current study, more medial than lateral meniscal repairs were performed $(26 \%$ vs. $19 \%$ ), with a total of $45 \%$ of patients undergoing concurrent meniscal repair. Patients $<20$ years $(39 \%)$ were significantly more likely to undergo concurrent medial meniscal repair than patients aged $20-30$ years $(21 \%)$ or $>30$ years (22\%).

Certain bony morphological characteristics of the proximal tibia and the distal femur have been associated with an increased risk for primary and recurrent ACL injuries [14, 15, 41, 45]. The PTS, as a surgically modifiable risk factor for ACL injuries, has attracted special attention in recent years. Clinical observations demonstrated a positive correlation between PTS and anterior tibial subluxation and rotatory knee laxity $[14,47]$. The mechanical impact of the PTS on ACL grafts has also been confirmed by several biomechanical studies, demonstrating increased ACL graft forces with increased PTS [4, 50]. Therefore, slope-reducing high tibial osteotomies have been proposed to counteract the negative effects of increased PTS on ACL grafts [9, 21, 51]. In this study, $13 \%$ of patients underwent concurrent osteotomies, with $40 \%$ of osteotomies representing slope-reducing osteotomies, indicating an increased awareness of the PTS as a potential cause of ACL graft failure. Consistent with previous reports, it was also shown that the LFCR is significantly higher in female compared to male patients [45], which may also be the reason for the significantly higher rate of contralateral ACL injuries in female patients.

Revision ACL-R has been shown to result in inferior patient reported outcomes (4-8 points less in Lysholm Score; 5-19 points less in Knee Injury and Osteoarthritis Outcome Score subscales) and increased residual laxity compared to primary ACL-R [13, 17, 37, 42, 53, 55, 56]. Independent modifiable predictors for inferior outcomes and higher failure rates after revision ACL-R compared to primary ACL-R have been identified and include graft choice and persistent rotatory knee laxity caused by increased PTS, loss of meniscal tissue, and peripheral capsuloligamentous insufficiency. This study demonstrated increased awareness of modifiable risk factors in revision ACL-R by an increasing number of concurrently performed procedures to address independent predictors of worse outcomes and thus reduce the risk of subsequent ACL graft failures. Future studies should elaborate on specific indications for concurrent surgical procedures to facilitate decision-making in revision ACL-R.

The retrospective nature of this study is associated with several limitations. Since most of the patients had their previous ACL-Rs performed in a hospital other than one of the two participating centers, it was not possible to report changes of intra-articular findings from primary ACL-R to most recent revision ACL-R. However, the main objective of this study was to evaluate trends in revision ACL-R, which was possible due to the large sample size. Including two centers and three experienced knee surgeons reduced selection bias and thus increases the generalizability of the presented data. Despite the observation of increasing complexity in revision ACL-R compared to previous reports, it is currently unknown how this affects clinical and functional outcomes, as patient reported outcomes were not collected in this study.

\section{Conclusions}

This study showed that quadriceps tendon autograft is becoming increasingly popular in revision ACL-R, especially in younger patients. In addition, a high and growing rate of concurrent meniscal repairs (45\%), LET (31\%), and osteotomies (13\%) was observed, underscoring the high surgical demands of revision ACL-R. Lateral extra-articular tenodesis was associated with intact cartilage and severely abnormal preoperative knee laxity, which represent indications for LET in revision ACL-R.

Author contributions All listed authors have contributed substantially to this work: PWW, VT, ST and LL: collected data, performed 
statistical analysis, literature review, and primary manuscript preparation; VM, AMJG, and BPL: assisted with interpretation of the results, initial drafting of the manuscript, as well as editing and final manuscript preparation. All authors read and approved the final manuscript.

Funding Open Access funding enabled and organized by Projekt DEAL.

\section{Compliance with ethical standards}

Conflict of interest VM reports educational grants, consulting fees, and speaking fees from Smith \& Nephew plc, educational grants from Arthrex, is a board member of the International Society of Arthroscopy, Knee Surgery and Orthopaedic Sports Medicine (ISAKOS), and deputy editor-in-chief of Knee Surgery, Sports Traumatology, Arthroscopy (KSSTA). In addition, VM has a patent Quantified injury diagnostics-U.S. Patent No. 9,949,684, Issued on April 24, 2018 issued to University of Pittsburgh. AMJG is a consultant for Smith \& Nephew plc. and reports research support from Smith \& Nephew plc. and Ossur.

Ethical approval This study was approved by the Institutional Review Boards of the University of Pittsburgh (No.: STUDY20050226) and the University of Western Ontario (No.: 101533).

Open Access This article is licensed under a Creative Commons Attribution 4.0 International License, which permits use, sharing, adaptation, distribution and reproduction in any medium or format, as long as you give appropriate credit to the original author(s) and the source, provide a link to the Creative Commons licence, and indicate if changes were made. The images or other third party material in this article are included in the article's Creative Commons licence, unless indicated otherwise in a credit line to the material. If material is not included in the article's Creative Commons licence and your intended use is not permitted by statutory regulation or exceeds the permitted use, you will need to obtain permission directly from the copyright holder. To view a copy of this licence, visit http://creativecommons.org/licenses/by/4.0/.

\section{References}

1. Alm L, Drenck TC, Frosch KH, Akoto R (2020) Lateral extraarticular tenodesis in patients with revision anterior cruciate ligament (ACL) reconstruction and high-grade anterior knee instability. Knee 27:1451-1457

2. Backhaus K, Erichson B, Plinke W, Weiber R (2016) Zeitreihenanalyse. In: Backhaus K, Erichson B, Plinke W, Weiber R (eds) Multivariate analysemethoden. Springer, Berlin, pp 125-161

3. Baratz ME, Fu FH, Mengato R (1986) Meniscal tears: the effect of meniscectomy and of repair on intraarticular contact areas and stress in the human knee. A preliminary report. Am J Sports Med 14:270-275

4. Bernhardson AS, Aman ZS, Dornan GJ, Kemler BR, Storaci HW, Brady AW et al (2019) Tibial slope and its effect on force in anterior cruciate ligament grafts: anterior cruciate ligament force increases linearly as posterior tibial slope increases. Am J Sports Med 47:296-302

5. Borchers JR, Kaeding CC, Pedroza AD, Huston LJ, Spindler KP, Wright RW (2011) Intra-articular findings in primary and revision anterior cruciate ligament reconstruction surgery: a comparison of the MOON and MARS study groups. Am J Sports Med 39:1889-1893
6. Brophy RH, Haas AK, Huston LJ, Nwosu SK, MARS Group, Wright RW (2015) Association of meniscal status, lower extremity alignment, and body mass index with chondrosis at revision anterior cruciate ligament reconstruction. Am J Sports Med 43:1616-1622

7. Brophy RH, Wright RW, David TS, McCormack RG, Sekiya JK, Svoboda SJ et al (2012) Association between previous meniscal surgery and the incidence of chondral lesions at revision anterior cruciate ligament reconstruction. Am J Sports Med 40:808-814

8. Chen JL, Allen CR, Stephens TE, Haas AK, Huston LJ, Wright RW et al (2013) Differences in mechanisms of failure, intraoperative findings, and surgical characteristics between single- and multiple-revision ACL reconstructions: a MARS cohort study. Am J Sports Med 41:1571-1578

9. Dejour D, Saffarini M, Demey G, Baverel L (2015) Tibial slope correction combined with second revision ACL produces good knee stability and prevents graft rupture. Knee Surg Sports Traumatol Arthrosc 23:2846-2852

10. Farr J, Tabet SK, Margerrison E, Cole BJ (2014) Clinical, radiographic, and histological outcomes after cartilage repair with particulated juvenile articular cartilage: a 2-year prospective study. Am J Sports Med 42:1417-1425

11. Getgood A, Brown C, Lording T, Amis A, Claes S, Geeslin A et al (2019) The anterolateral complex of the knee: results from the International ALC Consensus Group Meeting. Knee Surg Sports Traumatol Arthrosc 27:166-176

12. Getgood AMJ, Bryant DM, Litchfield R, Heard M, McCormack RG, Rezansoff A et al (2020) Lateral extra-articular tenodesis reduces failure of hamstring tendon autograft anterior cruciate ligament reconstruction: 2-year outcomes from the STABILITY study randomized clinical trial. Am J Sports Med 48:285-297

13. Grassi A, Ardern CL, Marcheggiani Muccioli GM, Neri MP, Marcacci M, Zaffagnini S (2016) Does revision ACL reconstruction measure up to primary surgery? A meta-analysis comparing patient-reported and clinician-reported outcomes, and radiographic results. Br J Sports Med 50:716-724

14. Grassi A, Macchiarola L, Urrizola Barrientos F, Zicaro JP, Costa Paz M, Adravanti P et al (2019) Steep posterior tibial slope, anterior tibial subluxation, deep posterior lateral femoral condyle, and meniscal deficiency are common findings in multiple anterior cruciate ligament failures: an MRI case-control study. Am J Sports Med 47:285-295

15. Grassi A, Signorelli C, Urrizola F, Macchiarola L, Raggi F, Mosca $M$ et al (2019) Patients with failed anterior cruciate ligament reconstruction have an increased posterior lateral tibial plateau slope: a case-controlled study. Arthroscopy 35:1172-1182

16. Grassi A, Zicaro JP, Costa-Paz M, Samuelsson K, Wilson A, Zaffagnini $S$ et al (2020) Good mid-term outcomes and low rates of residual rotatory laxity, complications and failures after revision anterior cruciate ligament reconstruction (ACL) and lateral extraarticular tenodesis (LET). Knee Surg Sports Traumatol Arthrosc 28:418-431

17. Hamrin Senorski E, Svantesson E, Baldari A, Ayeni OR, Engebretsen L, Franceschi F et al (2019) Factors that affect patient reported outcome after anterior cruciate ligament reconstruction-a systematic review of the Scandinavian knee ligament registers. $\mathrm{Br}$ J Sports Med 53:410-417

18. Herbst E, Albers M, Burnham JM, Fu FH, Musahl V (2017) The anterolateral complex of the knee. Orthop J Sports Med 5:2325967117730805

19. Herbst E, Albers M, Burnham JM, Shaikh HS, Naendrup JH, Fu FH et al (2017) The anterolateral complex of the knee: a pictorial essay. Knee Surg Sports Traumatol Arthrosc 25:1009-1014

20. Hoser C, Fink C, Brown C, Reichkendler M, Hackl W, Bartlett J (2001) Long-term results of arthroscopic partial lateral 
meniscectomy in knees without associated damage. J Bone Jt Surg Br 83:513-516

21. Imhoff FB, Mehl J, Comer BJ, Obopilwe E, Cote MP, Feucht MJ et al (2019) Slope-reducing tibial osteotomy decreases ACL-graft forces and anterior tibial translation under axial load. Knee Surg Sports Traumatol Arthrosc 27:3381-3389

22. Lind M, Menhert F, Pedersen AB (2012) Incidence and outcome after revision anterior cruciate ligament reconstruction: results from the Danish registry for knee ligament reconstructions. Am J Sports Med 40:1551-1557

23. Magnussen RA, Trojani C, Granan LP, Neyret P, Colombet P, Engebretsen L et al (2015) Patient demographics and surgical characteristics in ACL revision: a comparison of French, Norwegian, and North American cohorts. Knee Surg Sports Traumatol Arthrosc 23:2339-2348

24. MARS Group (2014) Effect of graft choice on the outcome of revision anterior cruciate ligament reconstruction in the Multicenter ACL Revision Study (MARS) Cohort. Am J Sports Med 42:2301-2310

25. MARS Group (2016) Meniscal and articular cartilage predictors of clinical outcome after revision anterior cruciate ligament reconstruction. Am J Sports Med 44:1671-1679

26. MARS Group (2020) Meniscal repair in the setting of revision anterior cruciate ligament reconstruction: results from the MARS cohort. Am J Sports Med 48:2978-2985

27. MARS Group (2020) Predictors of clinical outcome following revision anterior cruciate ligament reconstruction. J Orthop Res 38:1191-1203

28. MARS Group (2019) Rehabilitation predictors of clinical outcome following revision ACL reconstruction in the MARS cohort. J Bone Jt Surg Am 101:779-786

29. MARS Group (2019) Relationship between sports participation after revision anterior cruciate ligament reconstruction and 2-year patient-reported outcome measures. Am J Sports Med 47:2056-2066

30. Allen CR, Anderson AF, Cooper DE, DeBerardino TM, Dunn WR, MARS Group et al (2017) Surgical predictors of clinical outcomes after revision anterior cruciate ligament reconstruction. Am J Sports Med 45:2586-2594

31. Brophy RH, Wright RW, Huston LJ, Haas AK, Nwosu SK, MARS Group et al (2020) Rate of infection following revision anterior cruciate ligament reconstruction and associated patient- and surgeon- dependent risk factors: retrospective results from MOON and MARS data collected from 2002 to 2011. J Orthop Res. https://doi.org/10.1002/jor.24871

32. Cooper DE, Dunn WR, Huston LJ, Haas AK, Spindler KP, MARS Group et al (2018) Physiologic preoperative knee hyperextension is a predictor of failure in an anterior cruciate ligament revision cohort: a report from the MARS Group. Am J Sports Med 46:2836-2841

33. Ding DY, Zhang AL, Allen CR, Anderson AF, Cooper DE, MARS Group et al (2017) Subsequent surgery after revision anterior cruciate ligament reconstruction: rates and risk factors from a multicenter cohort. Am J Sports Med 45:2068-2076

34. Magnussen RA, Borchers JR, Pedroza AD, Huston LJ, Haas AK, MARS Group et al (2018) Risk factors and predictors of significant chondral surface change from primary to revision anterior cruciate ligament reconstruction: a MOON and MARS cohort study. Am J Sports Med 46:557-564

35. Wright RW, Huston LJ, Haas AK, Allen CR, Anderson AF, MARS Group et al (2019) Predictors of patient-reported outcomes at 2 years after revision anterior cruciate ligament reconstruction. Am J Sports Med 47:2394-2401

36. Wright RW, Huston LJ, Spindler KP, Dunn WR, Haas AK, MARS Group et al (2010) Descriptive epidemiology of the
Multicenter ACL Revision Study (MARS) cohort. Am J Sports Med 38:1979-1986

37. Mohan R, Webster KE, Johnson NR, Stuart MJ, Hewett TE, Krych AJ (2018) Clinical outcomes in revision anterior cruciate ligament reconstruction: a meta-analysis. Arthroscopy 34:289-300

38. Spindler KP, Huston LJ, Zajichek A, Reinke EK, Amendola A, MOON Knee Group et al (2020) Anterior cruciate ligament reconstruction in high school and college-aged athletes: does autograft choice influence anterior cruciate ligament revision rates? Am J Sports Med 48:298-309

39. Morgan JA, Dahm D, Levy B, Stuart MJ, MARS Group (2012) Femoral tunnel malposition in ACL revision reconstruction. J Knee Surg 25:361-368

40. Musahl V, Citak M, O'Loughlin PF, Choi D, Bedi A, Pearle AD (2010) The effect of medial versus lateral meniscectomy on the stability of the anterior cruciate ligament-deficient knee. Am J Sports Med 38:1591-1597

41. Napier RJ, Garcia E, Devitt BM, Feller JA, Webster KE (2019) Increased radiographic posterior tibial slope is associated with subsequent injury following revision anterior cruciate ligament reconstruction. Orthop J Sports Med 7:2325967119879373

42. Ouillette R, Edmonds E, Chambers H, Bastrom T, Pennock A (2019) Outcomes of revision anterior cruciate ligament surgery in adolescents. Am J Sports Med 47:1346-1352

43. Parker BR, Hurwitz S, Spang J, Creighton R, Kamath G (2016) Surgical trends in the treatment of meniscal tears: analysis of data from the american board of orthopaedic surgery certification examination database. Am J Sports Med 44:1717-1723

44. Persson A, Fjeldsgaard K, Gjertsen JE, Kjellsen AB, Engebretsen $\mathrm{L}$, Hole RM et al (2014) Increased risk of revision with hamstring tendon grafts compared with patellar tendon grafts after anterior cruciate ligament reconstruction: a study of 12,643 patients from the Norwegian Cruciate Ligament Registry, 2004-2012. Am J Sports Med 42:285-291

45. Pfeiffer TR, Burnham JM, Hughes JD, Kanakamedala AC, Herbst E, Popchak A et al (2018) An increased lateral femoral condyle ratio is a risk factor for anterior cruciate ligament injury. $\mathrm{J}$ Bone Jt Surg Am 100:857-864

46. Rahardja R, Zhu M, Love H, Clatworthy MG, Monk AP, Young SW (2020) Effect of graft choice on revision and contralateral anterior cruciate ligament reconstruction: results from the New Zealand ACL Registry. Am J Sports Med 48:63-69

47. Rahnemai-Azar AA, Abebe ES, Johnson P, Labrum J, Fu FH, Irrgang JJ et al (2017) Increased lateral tibial slope predicts highgrade rotatory knee laxity pre-operatively in ACL reconstruction. Knee Surg Sports Traumatol Arthrosc 25:1170-1176

48. Redler A, Iorio R, Monaco E, Puglia F, Wolf MR, Mazza D et al (2018) Revision anterior cruciate ligament reconstruction with hamstrings and extra-articular tenodesis: a mid- to long-term clinical and radiological study. Arthroscopy 34:3204-3213

49. Runer A, Csapo R, Hepperger C, Herbort M, Hoser C, Fink C (2020) Anterior cruciate ligament reconstructions with quadriceps tendon autograft result in lower graft rupture rates but similar patient-reported outcomes as compared with hamstring tendon autograft: a comparison of 875 patients. Am J Sports Med 48:2195-2204

50. Samuelsen BT, Aman ZS, Kennedy MI, Dornan GJ, Storaci HW, Brady AW et al (2020) Posterior medial meniscus root tears potentiate the effect of increased tibial slope on anterior cruciate ligament graft forces. Am J Sports Med 48:334-340

51. Sonnery-Cottet B, Mogos S, Thaunat M, Archbold P, Fayard JM, Freychet B et al (2014) Proximal tibial anterior closing wedge osteotomy in repeat revision of anterior cruciate ligament reconstruction. Am J Sports Med 42:1873-1880 
52. Spindler KP (2007) The Multicenter ACL Revision Study (MARS): a prospective longitudinal cohort to define outcomes and independent predictors of outcomes for revision anterior cruciate ligament reconstruction. J Knee Surg 20:303-307

53. Spindler KP, Huston LJ, Wright RW, Kaeding CC, Marx RG, Amendola A et al (2011) The prognosis and predictors of sports function and activity at minimum 6 years after anterior cruciate ligament reconstruction: a population cohort study. Am J Sports Med 39:348-359

54. Thygesen LC, Ersbøll AK (2014) When the entire population is the sample: strengths and limitations in register-based epidemiology. Eur J Epidemiol 29:551-558

55. Weiler A, Schmeling A, Stöhr I, Kääb MJ, Wagner M (2007) Primary versus single-stage revision anterior cruciate ligament reconstruction using autologous hamstring tendon grafts: a prospective matched-group analysis. Am J Sports Med 35:1643-1652

56. Wright R, Spindler K, Huston L, Amendola A, Andrish J, Brophy $\mathrm{R}$ et al (2011) Revision ACL reconstruction outcomes: MOON cohort. J Knee Surg 24:289-294

57. Wright RW, Dunn WR, Amendola A, Andrish JT, Bergfeld J, Kaeding CC et al (2007) Risk of tearing the intact anterior cruciate ligament in the contralateral knee and rupturing the anterior cruciate ligament graft during the first 2 years after anterior cruciate ligament reconstruction: a prospective MOON cohort study. Am J Sports Med 35:1131-1134

58. Wright RW, Magnussen RA, Dunn WR, Spindler KP (2011) Ipsilateral graft and contralateral ACL rupture at 5 years or more following ACL reconstruction: a systematic review. J Bone Jt Surg Am 93:1159-1165

59. Yoon KH, Kim JH, Kwon YB, Kim EJ, Kim SG (2020) Rerevision anterior cruciate ligament reconstruction showed more laxity than revision anterior cruciate ligament reconstruction at a minimum 2-year follow-up. Knee Surg Sports Traumatol Arthrosc 28:1909-1918

Publisher's Note Springer Nature remains neutral with regard to jurisdictional claims in published maps and institutional affiliations. 